\title{
Are Community Health Center Patients Interested in Self-Measured Blood Pressure Monitoring (SMBP) - And Can They Do It?
}

This article was published in the following Dove Press journal: Integrated Blood Pressure Control

\author{
Debosree Roy' \\ Margaret Meador ${ }^{2}$ \\ Nana Sasu ${ }^{2}$ \\ Kate Whelihan' \\ Joy H Lewis'
}

'School of Osteopathic Medicine in Arizona, A.T. Still University, Mesa, Arizona, USA; ${ }^{2}$ National Association of Community Health Centers, Bethesda, MD, USA
Correspondence: Debosree Roy School of Osteopathic Medicine in Arizona, A.T. Still University

Email debosreeroy@atsu.edu
Introduction: Self-measured blood pressure monitoring (SMBP) helps diagnose and manage hypertension from outside the clinic, which has implications for patient empowerment and outcomes, continuity of care, and resilience in care communities catering to vulnerable populations.

Methods: We instituted a protocol for SMBP among hypertensive patients at 9 community health centers in 3 states and administered questionnaires to patients before and after the protocol was instituted to assess knowledge and engagement with disease management, beliefs and attitudes towards, and experience doing SMBP. Questionnaires included 16 items designed to evaluate patient perceptions and beliefs about SMBP. These included a series of questions using a 5-point Likert scale, binary questions related to their perceived ability to comply with specific SMBP guidelines and open-ended questions to obtain descriptions of experiences with SMBP.

Results: The pre-questionnaire was completed by 478 patients and the post-questionnaire was completed by 372 . Seventy-seven percent of respondents knew their ideal blood pressure and their engagement with blood pressure management increased significantly $(p=0.0024)$ after completing the protocol. Additionally, $85 \%$ of respondents said that they had a positive experience doing SMBP. Open-ended responses revealed insight regarding why patients chose to do SMBP and factors patients appreciated about SMBP.

Discussion: When trained properly and supported, community health center patients are capable of and motivated to perform accurate SMBP. Our study provides evidence that health center patients can follow detailed SMBP protocols and monitor their own blood pressure from the safety of their homes, which is critical to their care continuum, particularly in days of a pandemic.

Keywords: SMBP, self-measured blood pressure monitoring, home blood pressure monitoring, patient engagement, community health centers

\section{Introduction}

Optimal outcomes in hypertension management can result from a comprehensive approach that includes strategies such as patient education, patient-provider symmetry in treatment goals and beliefs, ${ }^{1}$ and patient engagement in their own care. ${ }^{2,3}$ Patient education in self-management of chronic conditions should empower and equip patients to detect challenges in the care pathway and find solutions to overcome those challenges. ${ }^{4}$ These include empowering patients to develop confidence in making lifestyle and behavioral changes for improving outcomes. ${ }^{1}$ Self-measured blood pressure monitoring 
(SMBP), also known as home blood pressure monitoring, is the regular measurement of blood pressure by a patient outside the clinical setting. ${ }^{5}$ The use of SMBP can provide patient education opportunities and increase patient activation or engagement. ${ }^{6}$ SMBP also allows for patient-generated data to support hypertension management. SMBP is more predictive than office readings to diagnose hypertension and yields more accurate and complete data to manage hypertension. ${ }^{7,8}$ Recent clinical evidence suggests that, compared to usual care, SMBP along with clinical support, including individual counseling, patient education, and outreach, may enhance blood pressure control. $^{9-11}$

Studies also show that patients reliably report selfmeasured blood pressure readings ${ }^{12}$ and SMBP can improve medication adherence by empowering people to take a more active role in managing their blood pressure. ${ }^{13}$ Recent clinical guidelines such as those from the 7th Joint National Committee, recommend the use of SMBP for diagnosis and management of hypertension. ${ }^{14}$ Even so, not all providers are ready to endorse SMBP fully. Some have expressed concern about SMBP causing problems for them or their patients, ${ }^{15}$ such as yielding inaccurate or inconsistent readings. Previous research indicates that providers can be pessimistic about patients' abilities to successfully complete SMBP. ${ }^{16}$ Literature also suggests some providers have preferred ambulatory blood pressure management over home-blood pressure monitoring. ${ }^{15,17}$ This preference for ambulatory blood pressure management as their out-of-office method of choice may be related to the fact that it does not rely on patients having an active role in generating measurements. However, SMBP is better tolerated, less expensive, and more easily accessible than ambulatory blood pressure management systems. ${ }^{18}$ Importantly, provider recommendation is a key reason patients do SMBP, ${ }^{19}$ which augments the need for clinicians to be encouraged to support and promote SMBP.

To optimize efficacy of patient-centered disease management, interventions must be studied from the perspective of patients, especially those who are served by community health centers (CHCs). CHCs are community based, patient directed organizations which provide comprehensive primary care irrespective of the patients' insurance status or ability to pay for service; these patients are often the most vulnerable. It is important to capture patient perspectives from underserved populations, related to processes and procedures that depend on their active participation and engagement. While prior research supports patients reliably reporting home blood pressure measurements to their care team, ${ }^{12}$ patient perceptions about SMBP when undertaken with training, education, outreach, and other supports has not been explored in underserved populations served by CHCs. Research shows strong evidence for SMBP in controlling blood pressure, but only when combined with support activities such as patient education and training, reminders, and a systematic way to receive and use data for clinical decision-making. ${ }^{10,20}$ While general assessments about ability to perform SMBP have been conducted, we found no studies that examine patient perceptions regarding their ability to follow each recommended step for taking accurate and consistent measurements. ${ }^{16}$

Although SMBP has been shown to be accepted in some patient populations, ${ }^{21}$ other research has shown that patients are skeptical of home BP measurements because of uncertainty over device reliability or technique. ${ }^{16}$ Patients in a previous study were asked to watch an instructional web-based video at home and received no additional training or education amounting to inadequate training for conducting SMBP. ${ }^{16}$ Data from British clinical trials show that with adequate training, patients feel confident in their ability to do SMBP and feel that home blood pressure readings were more accurate than office measurements. $^{22}$ Understanding the opportunities, challenges, and barriers for $\mathrm{CHC}$ patients to generate their own clinically relevant data is an important step in facilitating increased acceptance and uptake of SMBP and improving hypertension outcomes.

The objective of this study was to gather information related to whether patients were adept at, were willing to and were able to do SMBP, and how they liked doing SMBP. Health centers wished to determine if they could implement SMBP with their patients in order to generate reliable data for clinical decision-making in managing hypertension.

The specific aims of this study were:

1. To assess patient knowledge about hypertension and engagement in their own care

2. To assess patient attitudes and belief towards SMBP before and after doing SMBP

3. To assess patient experience doing SMBP.

\section{Methods}

Nine community health centers in Kentucky, Missouri, and New York participated in a project sponsored by the National Association of Community Health Centers (NACHC) to accelerate use of SMBP. Health centers were invited to participate in the project based on their 
geographic alignment with Y-USA (who provided health behavior modification services). Also, those invited had engagement from the local health department on supporting SMBP. $^{22}$ In the initiative, health center care teams trained patients with uncontrolled hypertension to use a home blood pressure monitor, accurately take their own blood pressure, understand their blood pressure goals and readings, and return the blood pressure readings to their care team as a part of newly implemented SMBP care models. The development of these SMBP care models was part of a larger goal to leverage clinical-community collaborations as an approach for decreasing uncontrolled blood pressure in community health center populations. ${ }^{23}$ For standardization, completing a cycle of SMBP was defined as performing self-measurement of blood pressure at home, morning and evening, for three consecutive days.

Criteria for inclusion and exclusion in being offered SMBP varied slightly across participating locations; however, the basic clinical criteria (age, elevated BP in the period before protocol commencement and a diagnosis of hypertension) were consistent across all locations. One location in Kentucky assessed patient literacy and numeracy skills before enrollment and a health center in Missouri excluded patients whose primary language was not English or Spanish, unless the patient had a translator who could help them with the protocol and associated processes. This health center also excluded patients with a recent cardiac event, evidence of heart rhythm problems or risk for lymphedema.

Patients were identified through pre-visit planning, including care team huddles and registries. During a visit, patients were recommended to use SMBP. If the patient consented to take part in the protocol, they were trained by a care team member, eg, nurse, medical assistant, pharmacist, community health worker, to use a home blood pressure monitor. The patient was then given or loaned a home blood pressure monitor.

While doing SMBP, patients were offered support through outreach by their care team or the health center's collaboration partner (eg, local health department nurses, local YMCAs, Americorps volunteers). After monitoring their blood pressure for a period of time determined by their clinician (ranging from one week to one month), patients returned to their health center, where their blood pressure measurements were averaged and assessed; patients then received medication titration and healthy lifestyle recommendations as appropriate.
In October 2017, after all participating health center teams developed their SMBP care models, NACHC introduced preand post-SMBP patient questionnaires. Training on the questionnaires was provided to health center care teams via teleconference/webinar, after which, care teams implemented the questionnaire in their clinics. Questionnaires were available to patients in both digital and paper formats. Responses to the electronic-based questionnaire were collected using Qualtrics software (C) 2020 (Provo, Utah). Paper questionnaires were administered by the health care team and, when complete, sent via secured fax or email to NACHC. Each patient was assigned an anonymized participant ID based on a combination of the blood pressure monitor serial number and a health center code. The anonymized IDs were noted on the pre- and post- questionnaires. Patients were encouraged to complete the questionnaires on their own. However, staff supported their completion depending on patient literacy and ability. The questionnaires were available in seven languages, including Arabic, Dari, Haitian Creole, Spanish, Swahili, Somali, and English. All patients could refuse to complete any question or questionnaire. The protocols ran from October 2017 to June 2018. Questionnaires were administered individually to patients before and after they participated in the protocol.

The SMBP process is briefly outlined in Figure 1.

\section{Questionnaires \\ Pre-Questionnaire}

The pre-questionnaire included 16 items designed to evaluate patient perceptions and beliefs about the use of home blood pressure monitoring as a tool to help control their blood pressure. In the questionnaire, the patient selected the type of monitor they were using and were asked to provide a short, written response about their reason for doing SMBP. Patients were also asked about their understanding of their blood pressure goal and given an opportunity to write down the goal if they indicated they were aware of the target numbers. The pre-questionnaire assessed whether patients knew what to do if their blood pressure readings at home were too high. The questionnaire also assessed whether and to what degree they were responsible for managing their blood pressure as indicators of their level of hypertension education and engagement in their own care. The pre-questionnaire also included a series of questions that used 5-point Likert scale (Strongly Agree $=5$ to Strongly Disagree=1) response options. Five questions measured patients' beliefs about their responsibility and power in managing their blood 


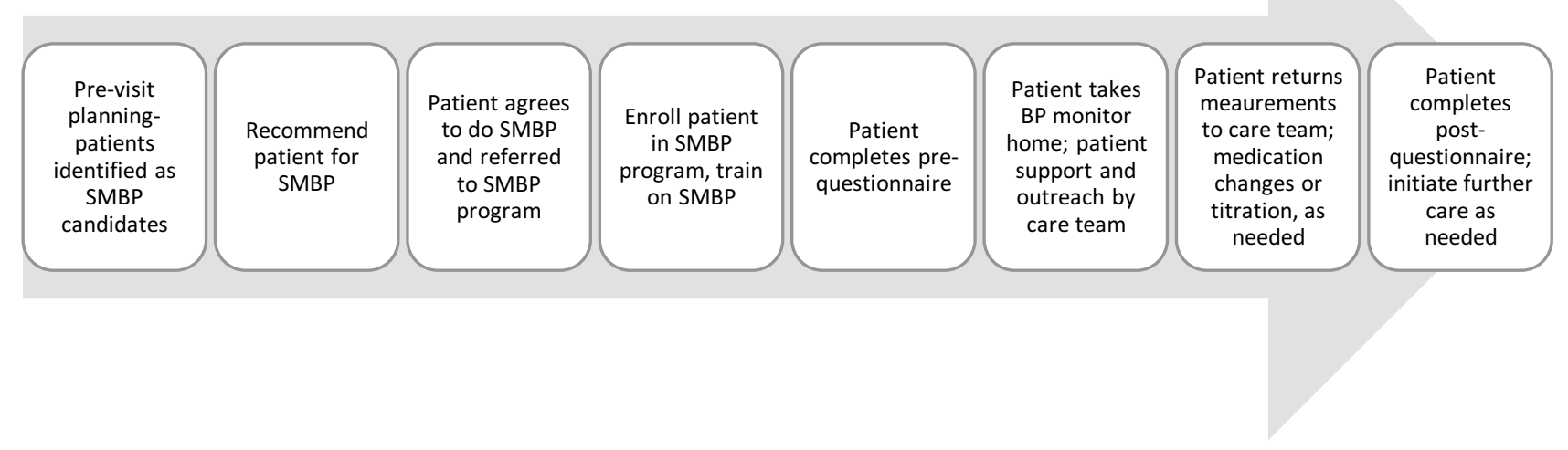

Figure I Work flow process of SMBP.

pressure and their confidence and comfortability in using a home blood pressure monitor. Two questions focused on self-efficacy in completing the steps to prepare to take blood pressure measurements outside of the clinical setting and then return those readings to the care team. Demographic information was also collected.

\section{Post-Questionnaire}

The post-questionnaire included 20 items designed to understand the patients' overall experience with SMBP, including if they would recommend SMBP to someone they knew. Many questions from the pre-questionnaire were repeated to determine if there were any differences in responses after patients participated in SMBP, as a way to compare their expectations about SMBP with their actual experience. Patients responded to a 5-point Likert scale (Very Good $=5$ to Very Poor $=1$ ) on their experience and were asked to provide a written reason for their answer. Patients were re-asked four questions from the pre-questionnaire assessing beliefs about their responsibility and power in managing their blood pressure and their ability to use the home blood pressure monitor. The post-questionnaire also re-asked self-efficacy questions from the prequestionnaire to gauge patients' actual abilities to complete SMBP compared to their expectations. These included key behaviors important in producing accurate SMBP measurements, such as avoiding exercise, alcohol, caffeine, cold medicines, and eating for 30 minutes before doing SMBP, resting for 5 minutes before doing SMBP, avoiding TV while doing SMBP, and sitting while doing SMBP. Finally, patients were given an opportunity to reflect on any positive or negative experiences with SMBP. The post-questionnaire did not include questions related to demographic information. The pre- and post-questionnaires are included as Appendices. Both questionnaires were evaluated by stakeholders for face validity and pilot-tested in a sample of patients before they were administered to the broader population.

\section{Data Analysis Quantitative Analysis}

We used summary statistics (frequency/percentage) to describe the population characteristics of SMBP patients and assess their knowledge of hypertension management. We also used descriptive statistics and Wilcoxon signedrank tests to compare responses between patients who completed the pre questionnaire and those who completed both the pre and post questionnaires. Responses from patients who only completed the post questionnaire were not evaluated due to the lack of data points to measure change in attitude and beliefs from before and after administration of protocol. Items evaluated were patients' ability to carry out preparatory steps associated with SMBP and their attitudes and beliefs about hypertension management and SMBP. Respondents with responses to at least one question from both questionnaires were retained for data analysis assessing changes in responses between the pre and post questionnaires. The objective of such a comparison was to assess summative changes in the items evaluated in the same population before and after administration of the protocol. Data were analyzed with SAS version 9.4 (Cary, NC).

\section{Qualitative Analysis}

There were three open-ended questions used in the qualitative analysis-one from the pre-questionnaire and two in the post-questionnaire. These questions focused on 
understanding patient expectations of, beliefs about, and experiences with SMBP. All open-ended questionnaire responses, electronic- and paper-based, were collated into a single file and analyzed using NVIVO 12 (QSR International Pvt. Ltd.) to determine high frequency words and phrases. From these results, we developed a codebook to identify and categorize emerging patterns and themes.

The study was determined to be exempt by the Institutional Review Board of A.T. Still University's Arizona campus (IRB protocol \#2017-033) because extant patient data underwent a de-identification process by persons with routine access to patient records before transmission to study investigators. Information collected prospectively was recorded by investigators in such a manner that subjects could not be identified, directly or through identifiers linked to the subjects.

\section{Results}

\section{Demographic Characteristics}

There were 478 patients who completed the prequestionnaire. Three hundred and seventy-two patients completed the post questionnaire; 279 patients completed both a pre- and post-questionnaire during the project period (Figure 2). The demographic characteristics of patients who completed the pre-questionnaire and both pre- and post-questionnaires are provided in Table 1 .

\section{Quantitative Analysis}

Patient Knowledge About and Engagement in Their Hypertension Management

Patient response to one question in the pre-questionnaire about blood pressure goals (BP) as an indicator of patient education/knowledge level about their hypertension is provided in Table 2.

We also assessed patient engagement in their hypertension management by asking them both before and after using SMBP whether they believed they were the person most responsible for managing their blood pressure. The assessment is presented in Table 3 .

\section{Attitudes and Belief About SMBP}

Patient assessments of their ability to use a home blood pressure monitor without trouble and ability to get blood pressure readings to their health care team are provided in Table 4.

\section{Support During SMBP}

When patients were asked about their level of comfort asking their care team for help with SMBP in the prequestionnaire, patients noted that they were very comfortable or comfortable $(89.57 \%)$.

\section{Expectations and Experience Preparing for SMBP}

Expectations about and actual experience with being able to prepare for SMBP were compared between two groups (pre and those who completed both the pre and post questionnaires). Results are presented in Table 5.

\section{Experience Doing SMBP}

Additional items in the post questionnaire assessed patient experience with SMBP using 5-point Likert scales; responses were collapsed to a 3-point Likert-like scale for ease of presentation. These are presented in Table 6 .

\section{Qualitative Analysis}

\section{Attitudes and Beliefs About SMBP}

The pre-questionnaire included one open ended question that asked patients why they decided to do SMBP. There were 364 responses (76.15\% response rate), including 35 translated from Spanish. Responses were categorized by theme, with some responses identified under multiple themes. The most frequently volunteered reason patients provided for doing SMBP was because their doctors recommended it $(n=168)$. Some patients responded with brief statements like "high blood pressure" or "control high blood pressure" $(\mathrm{n}=104)$. However, many patients reported deciding to do SMBP due to intrinsic motivational factors, such as to improve their health or out of concern for their health $(n=28)$. Others indicated they decided to try SMBP to participate more in their own healthcare and recognized SMBP as a tool to help them

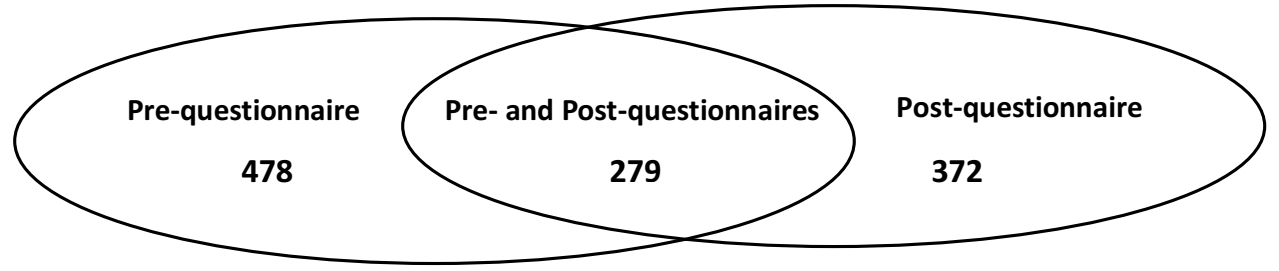

Figure 2 Distribution of pre-, pre- and post-, and post-questionnaire. 
Table I Demographic Characteristics of Patients

\begin{tabular}{|l|l|l|l|l|}
\hline Demographics & \multicolumn{2}{|l|}{$\begin{array}{l}\text { Respondents } \\
\text { to Pre-Survey } \\
\text { (n=478) }\end{array}$} & \multicolumn{2}{l|}{$\begin{array}{l}\text { Respondents } \\
\text { to Pre and } \\
\text { Post (n=279) }\end{array}$} \\
\hline Sex & $\mathbf{n}$ & $\%$ & $\mathbf{n}$ & $\%$ \\
\hline Male & 225 & 47.07 & 129 & 46.4 \\
Female & 243 & 50.84 & 147 & 52.88 \\
Other & 1 & 0.21 & 1 & 0.36 \\
Unknown/Missing & 9 & 1.88 & 2 & 0.36 \\
\hline Age & & & & \\
<34 years & 52 & 10.88 & 24 & 8.66 \\
35-44 years & 97 & 20.29 & 50 & 18.05 \\
45-54 years & 124 & 25.94 & 75 & 27.08 \\
55-64 years & 117 & 24.48 & 75 & 27.08 \\
65 years and older & 76 & 15.90 & 50 & 18.05 \\
Unknown/Missing & 12 & 2.51 & 3 & 1.00 \\
\hline Race & & & & \\
White & 315 & 65.90 & 179 & 65.81 \\
Black/African American & 87 & 18.20 & 51 & 18.75 \\
Asian & 7 & 1.46 & 5 & 1.84 \\
American Indian/Alaska Native & 5 & 1.05 & 0 & 0 \\
Other & 26 & 5.44 & 19 & 6.99 \\
Unknown/Missing & 38 & 7.95 & 25 & 8.00 \\
\hline Ethnicity & & & & \\
Hispanic/Latino & 56 & 11.72 & 45 & 16.30 \\
Non-Hispanic/Latino & 395 & 82.64 & 228 & 82.61 \\
Unknown/Missing & 27 & 5.65 & 6 & 2.00 \\
\hline
\end{tabular}

do that $(n=82)$. One comment was: "[I decided to do SMBP because] I want to stay informed about my daily fluctuations in BP to help bring it under control." Another person responded, "I thought it was a good thing to keep an eye on [my blood pressure] for myself."

Patients also indicated they decided to do SMBP because they believed it could be a way to help them control their blood pressure without medication. A number of patients gave reasons for trying SMBP such as, "[I have been taking] blood pressure meds for 2 years and can I be off?" Other patients believed that measurements taken at home would help provide more complete or accurate information about their blood pressure for treatment, eg, "I am currently on 3 meds. Hoping that regular BP readings will allow us to optimize dosages" and "In the office my numbers were all over the place."

Some patients stated they found motivation to make positive dietary and physical activity changes when they had access to their blood pressure readings. For example,
Table 2 Patients' Knowledge About Blood Pressure Goals, PreQuestionnaire

\begin{tabular}{|c|c|c|}
\hline \multicolumn{3}{|c|}{$\begin{array}{l}\text { Patients Who Stated They Knew Their Blood Pressure Goal } \\
\text { and Those Able to Write It }\end{array}$} \\
\hline & $\mathbf{n}$ & $\%$ \\
\hline Knew BP Goal & 368 & 76.99 \\
\hline Did not know BP Goal & 73 & 15.27 \\
\hline Unknown/Missing & 37 & 7.74 \\
\hline Able to Write BP goal & 341 & 71.34 \\
\hline \multicolumn{3}{|l|}{ BP goal systolic $\leq$ I 40} \\
\hline $110-120$ & 78 & 22.87 \\
\hline $121-130$ & 138 & 40.46 \\
\hline $13|-| 40$ & 121 & 35.48 \\
\hline \multicolumn{3}{|l|}{ BP goal diastolic $\leq 90$} \\
\hline $60-70$ & 10 & 2.93 \\
\hline $7 I-80$ & 193 & 56.59 \\
\hline $81-90$ & $|3|$ & $38.4 I$ \\
\hline
\end{tabular}

"it keeps you aware of how to manage you[r] lifestyle such as choosing food you eat and exercise." Patients were also more aware of specific behaviors that "triggered" or "spiked" their blood pressure, such a smoking (Table 7).

\section{Experience Doing SMBP}

Most patients responding to the post-questionnaire (311/ $367 ; 84.7 \%$ ) said that they had a positive experience participating in SMBP. Just over half of respondents $(164 / 311 ; 52.73 \%)$ provided an explanation for their experience rating. The most frequent explanation provided for positive experiences related to liking being empowered to monitor their blood pressure themselves and/or the ability to know their readings any time. Many patients indicating a positive experience also conveyed that SMBP was easy to do or that they learned about/gained awareness about their hypertension. (Table 8)

Several patients attributed their positive experience to the association between medication adherence and blood pressure control. For example, [SMBP] "gave me a picture of how well my medications are working," and,

I stopped my blood pressure med[ication] in hopes to control it on my own. I was staying in the red zone so I started my [blood pressure] med[ication] back - it is now controlled.

Patients also described being able to connect lifestyle behaviors with their blood pressure measurements or gaining insights about what triggers high readings as reasons 
Table 3 Patient Engagement in Hypertension Management

\begin{tabular}{|l|l|l|l|l|}
\hline \multirow{2}{*}{ Questions } & \multicolumn{2}{|l|}{$\begin{array}{l}\text { Pre- } \\
\text { Questionnaire }\end{array}$} & \multicolumn{2}{l|}{$\begin{array}{l}\text { Post- } \\
\text { Questionnaire** }\end{array}$} \\
\cline { 2 - 5 } & $\mathbf{n}$ & $\%$ & $\mathbf{n}$ & $\%$ \\
\hline $\begin{array}{l}\text { I am the person most } \\
\text { responsible for managing } \\
\text { my blood pressure* }\end{array}$ & & & & \\
Strongly agree & 209 & 75.45 & 218 & 78.14 \\
Agree & 37 & 13.36 & 50 & 17.92 \\
Undecided & 4 & 1.44 & 9 & 3.23 \\
Disagree & 3 & 1.08 & 0 & 0 \\
Strongly disagree & 24 & 8.66 & 2 & 0.72 \\
\hline
\end{tabular}

Notes: *Difference between pre and pre and post was statistically significant $(p=0.0024)$. **Responses from patients who responded to at least one question from both questionnaires were retained for analysis.

for their positive experience with SMBP. "[My experience was positive because I am] becoming aware of daily reading $[\mathrm{s}]$ and evaluating my food intake and exercise." Other reasons patients gave for their positive SMBP experience was feeling more comfortable at home and that they understood the instructions and felt adequately prepared to undertake the process by themselves. Patients who were able to follow the instructions also learned how to customize the experience to make it part of their everyday lives. "I was given instructions on how to use [the monitor] and I created a habit which has helped me."

Most patients who rated their experiences with SMBP as neutral or negative $(56 / 367,15.26 \%)$ attributed it to not having enough time or forgetting to check their blood pressure; another reason volunteered was blood pressure cuffs that did not fit comfortably or machines that had low batteries; one respondent indicated SMBP was "a hassle" and one expressed not remembering the directions once home. Despite some negative experiences, the overwhelming majority of patients (93\%) said they would recommend SMBP to others.

A final open-ended question asked patients to describe any other positive or negative things about monitoring their blood pressure at home. Most of these responses were positive and many had themes similar to those cited for the experience question, including easy to do, good to know own readings on a regular basis, learning and awareness about managing high blood pressure, and engagement in their own health. In addition, some patients who were using loaned home blood pressure monitors indicated they wanted to keep the monitor. Some patients who described difficulty incorporating SMBP into their schedule noted as
Table 4 Patients' Attitudes and Beliefs Before and After SMBP

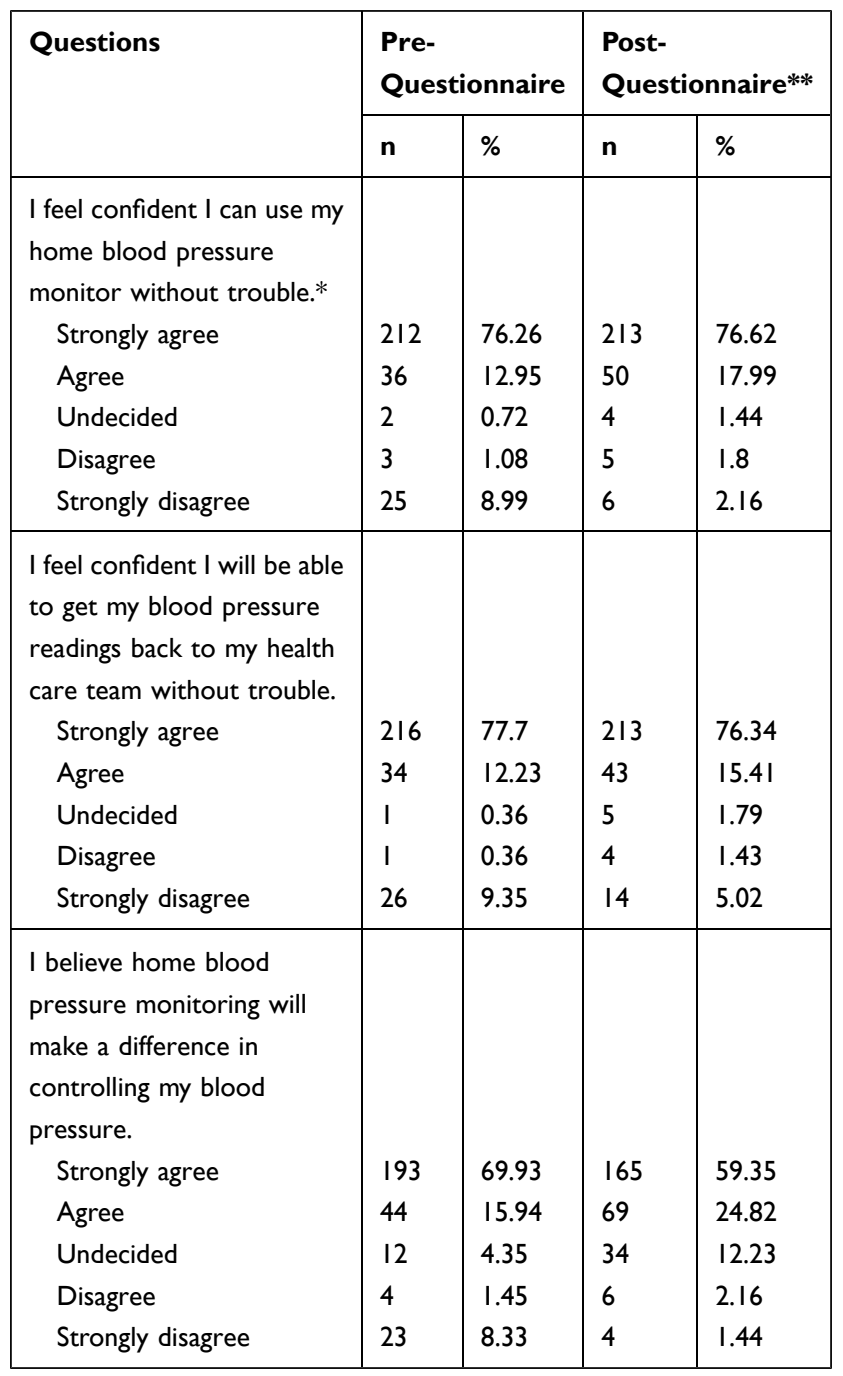

Notes: *Difference between pre and pre and post was statistically significant $(p=0.00494)$.**Responses from patients who responded to at least one question from both questionnaires were retained for analysis.

a response to the final question that they were able to find a way around obstacles to SMBP. Most negative responses to this question concerned difficulty putting on the cuff by themselves or cuff size being too small.

\section{Discussion}

Optimal patient outcomes depend on patients and providers having the same attitude and belief (symmetry) towards patient-directed management of diseases. ${ }^{1}$ Selfmeasured blood pressure monitoring is an evidence-based strategy shown to improve hypertension outcomes when combined with additional support, ${ }^{9-11}$ but relies on a patient-provider partnership. This partnership is underpinned by symmetry in the belief that patients are capable of and willing to perform accurate SMBP. Prior research 
Table 5 Patients' Expected vs Actual Ability to Carry Out Preparatory Steps Associated with SMBP

\begin{tabular}{|c|c|c|c|c|}
\hline \multirow[t]{2}{*}{ Questions } & \multicolumn{2}{|c|}{$\begin{array}{l}\text { Pre- } \\
\text { Questionnaire }\end{array}$} & \multicolumn{2}{|c|}{$\begin{array}{l}\text { Post- } \\
\text { Questionnaire** }\end{array}$} \\
\hline & $\mathbf{n}$ & $\%$ & $\mathbf{n}$ & $\%$ \\
\hline \multicolumn{5}{|l|}{ Avoid exercise } \\
\hline Yes & 255 & 92.06 & 253 & 90.68 \\
\hline No & 22 & 7.94 & 26 & 9.32 \\
\hline \multicolumn{5}{|l|}{ Avoid alcohol } \\
\hline Yes & 257 & 92.78 & 254 & 91.04 \\
\hline No & 20 & 7.22 & 25 & 8.96 \\
\hline \multicolumn{5}{|l|}{ Avoid caffeine } \\
\hline Yes & 234 & 84.48 & 227 & 81.36 \\
\hline No & 43 & 15.52 & 52 & 18.64 \\
\hline \multicolumn{5}{|l|}{ Avoid eating } \\
\hline Yes & 256 & 92.42 & 247 & 88.53 \\
\hline No & 21 & 7.58 & 32 & 11.47 \\
\hline \multicolumn{5}{|l|}{ Avoid medicine } \\
\hline Yes & 247 & 89.17 & 242 & 86.74 \\
\hline No & 30 & 10.83 & 36 & 12.90 \\
\hline \multicolumn{5}{|l|}{ Avoid watching TV } \\
\hline Yes & 241 & 87.00 & 241 & 87.00 \\
\hline No & 36 & 13.00 & 36 & 13.00 \\
\hline \multicolumn{5}{|l|}{ Able to sit } \\
\hline Yes & 257 & 92.78 & 246 & 88.81 \\
\hline No & 20 & 7.22 & 31 & 11.19 \\
\hline \multicolumn{5}{|l|}{ Able to rest for 5 minutes } \\
\hline Yes & 263 & 94.95 & 256 & 92.42 \\
\hline No & 13 & 4.69 & 20 & 7.22 \\
\hline
\end{tabular}

Notes: **Responses from patients who responded to at least one question from both questionnaires were retained for analysis.

has shown that some providers are unsure if patients are able to use SMBP effectively. ${ }^{15,16}$ We believe this perception may be even more pronounced among health center clinicians because of the social and economic barriers many safety net patients experience. While our study did not evaluate health center provider views, it examined the experience of health center patients with SMBP. Overwhelmingly, the results showed that health center patients can and want to do SMBP.

We know clinician recommendation for SMBP is a powerful factor in influencing patients to learn about and try SMBP. ${ }^{19}$ Results from our study confirmed this phenomenon, which has implications for future directions in provider education on shared decision making around SMBP. However, importantly, we also found that a substantial number of patients chose to do SMBP for intrinsic reasons, including wanting to get their blood pressure under control, improve their health, take a more active role in their hypertension management, and see if they could adjust lifestyle behaviors and adhere better to medication regimens. Some patients were hoping to improve enough to stop medication. Moreover, we found that more than $2 / 3$ of patients knew their systolic/diastolic blood pressure goals and most believed they were in control of their blood pressure management even before doing SMBP ( $88 \%$ responding to the pre questionnaire vs $90 \%$ responding to the pre and post questionnaire). These findings suggest that health center patients with hypertension are already engaged in their care to a certain degree and are ready partners for an intervention like SMBP. The idea that health center patients' attitudes and beliefs would posture them well for participating in home blood pressure monitoring is further reinforced by both the high levels of confidence patients expressed in whether they felt they could successfully prepare for SMBP and positive reports of their actual experience preparing for and carrying out SMBP. Most patients believed they could complete SMBP successfully, and they did.

Table 6 Patients' Experience with SMBP from the Post-Questionnaire

\begin{tabular}{|l|l|l|l|}
\hline Experience Doing SMBP $(\mathbf{n})^{*}$ & Positive & Neutral & Negative \\
\hline Doing SMBP in the morning (347) & $311(89.62 \%)$ & $18(5.19 \%)$ & $18(5.19 \%)$ \\
Doing SMBP at Night (349) & $310(88.82 \%)$ & $20(5.76 \%)$ & $19(5.48 \%)$ \\
Doing SMBP for recommended days (350) & $312(89.14 \%)$ & $16(4.61 \%)$ & $22(6.34 \%)$ \\
Ease of SMBP monitor use (350) & $319(91.14 \%)$ & $20(5.76 \%)$ & $11(3.17 \%)$ \\
Using the smartphone application to send readings (59) & $36(61.01 \%)$ & $2(0.58 \%)$ & $21(6.05 \%)$ \\
Record readings (348) & $322(92.52 \%)$ & $7(2.02 \%)$ & $19(5.48 \%)$ \\
Bring monitor to doctor visits (320) & $295(92.18 \%)$ & $7(2.02 \%)$ & $18(5.19 \%)$ \\
Bring SMBP log to doctor visits (330) & $296(89.69 \%)$ & $7(2.02 \%)$ & $24(6.92 \%)$ \\
\hline
\end{tabular}

Notes: *Total responses to each measure. Responses are not mutually exclusive. 
Table 7 Most Frequent Responses for Reasons Why Patients Did SMBP Reported in Post-Questionnaire $(\mathrm{n}=364)^{*}$

\begin{tabular}{|l|l|}
\hline Response & $\begin{array}{l}\text { Number of } \\
\text { Responses }\end{array}$ \\
\hline Doctor's recommendation & 168 \\
Statement of high blood pressure or to control/lower blood pressure & 104 \\
To better participate in their own healthcare (eg, monitoring regularly, knowing numbers at home, keeping an eye on/ & 82 \\
tracking BP, seeing if medications are working, seeing if lifestyle changes will lower BP) & 28 \\
To improve health/worried about health & 21 \\
Other (eg, headaches, free program, other incentive) & \\
\hline
\end{tabular}

Note: *Some responses have been coded into multiple categories.

Table 8 Patients' Explanation for Why They Liked Doing SMBP in Post-Questionnaire $(n=108)^{*}$

\begin{tabular}{|l|l|}
\hline Response & $\begin{array}{l}\text { Number of } \\
\text { Responses }\end{array}$ \\
\hline $\begin{array}{l}\text { Liked monitoring own BP/seeing readings at } \\
\text { home }\end{array}$ & 41 \\
Easy to do & 31 \\
Other positive (eg, good experience, was able to & 21 \\
do it, calmed me down, helpful, think all people & \\
should have a monitor, no malfunctions) & \\
Learning and awareness about their BP & 19 \\
Connected BP to lifestyle/other triggers & 15 \\
Associated with medication adherence & 7 \\
More comfortable at home & 5 \\
Understood instructions/prepared & 2 \\
\hline
\end{tabular}

Note: *Some responses have been coded into multiple categories.

We know out-of-office blood pressure measurement is superior to in-office measurement, both because it eliminates white coat effect and generates a pattern of readings over time ${ }^{7,8}$ - in essence, it yields better data for clinicians to make appropriate care decisions in treating hypertension. Our qualitative analysis reflected how impactful SMBP was on patient engagement in their blood pressure management, which is critical in achieving blood pressure control and reducing cardiovascular risk. Our findings show that SMBP positively influenced patients' awareness and understanding of hypertension, as well as their knowledge about the role of medications and lifestyle behaviors in determining blood pressure levels. Patients explicitly discussed the value of regular access to their blood pressure readings in seeing how medications, diet, and exercise are integral to helping control their blood pressure.

Importantly, our study showed that undergirding patients' ability to use SMBP successfully (and thereby reap the benefits of both better clinical information and increased engagement in their own care) are clinical supports. Health centers followed a protocol of training and orienting patients to their home blood pressure monitor as a critical initial step in SMBP procedures. This training included how to operate the device, techniques for preparation and positioning, how often to take measurements, and how they should send data to their care team. While implemented in different ways, every health center had outreach/reminder systems to connect with patients while they were doing SMBP. As has been shown in prior research, we believe that equipping patients with education and training and supporting them through the SMBP process was instrumental in patients' overwhelmingly positive responses to and successful use of SMBP. ${ }^{10}$

In view of the novel coronavirus pandemic, it has become quite apparent that health systems will need to engage patients more than ever in managing chronic conditions like hypertension. SMBP provides a unique opportunity for providers and patients alike to diagnose hypertension, keep track of hypertension disease and treatment outcomes, as well as titrate medications, while observing pandemic related public health practices like social distancing and shelter-at-home. In the post-pandemic world it is likely that telehealth and homebased care utilizing technology will continue to increase in importance.

\section{Limitations}

This study had some limitations regarding sampling and population selection. Patients identified as candidates for SMBP comprised a convenience sample of those patients with scheduled visits for hypertension during the study timeframe. Moreover, as mentioned elsewhere, protocol inclusion and exclusion criteria were not uniform across the health centers; some excluded patients who met the clinical criteria for SMBP but were not primarily English or Spanish speakers or lacked numerical skills. Patients were not compelled to respond to either the pre or post 
survey; thus, we do not have a complete linked set of pre/ post responses. Some patients only completed the presurvey, some only completed the post-survey, and a portion completed both. Clinical supports offered to patients (eg, training, education, and outreach), while universally implemented, were delivered differently by each health center. In addition, while care teams were instructed not to influence questionnaire responses, in some cases, they assisted patients in physically completing questionnaires, which could have influenced patient responses.

\section{Future Directions}

Future studies should explore ways to leverage this information to increase uptake of SMBP more broadly among health systems. More detailed exploration of the characteristics that differentiated patients who had positive vs negative experiences with SMBP is warranted. In particular, future studies should examine barriers to SMBP and oversample under-represented populations to see if there are any consistent challenges to their interest in and ability to conduct SMBP. Future research should also assess health outcomes of patients who participated in SMBP. Longitudinal studies would provide a better understanding of associated health outcomes as well as the effectiveness of SMBP procedures. One study found that patients start SMBP just to know their blood pressure; ${ }^{19}$ however what implications this has for continued use is unknown.

In June 2020, the American Heart Association and American Medical Association issued a joint policy statement that provided guidance for administering SMBP at home. ${ }^{24}$ We believe national standards will accelerate clinical use of SMBP and allow for related data elements to be systematized in electronic health records and incorporated into clinical quality metrics; these activities, in turn, would enable the kind of longitudinal studies required to optimize use of and better understand the clinical applications of SMBP.

\section{Conclusion}

When trained properly and supported, CHC patients are capable of and motivated to do accurate SMBP. In addition, while previous assessments have shown that patients are generally capable of returning home BP monitoring readings to their clinical team, ${ }^{12}$ our study examined patient perceptions regarding their ability to follow all recommended steps for taking accurate and consistent measurements that would yield clinically actionable patterns of data as well as getting these data to the care team.
This much more detailed analysis revealed that most patients had positive expectations for and experiences with SMBP, that it empowered them to self-manage, and that they would recommend it to others. Confirming that CHC patients can generate their own clinically relevant data is an important step in facilitating increased acceptance and uptake of SMBP and improving hypertension outcomes.

\section{Acknowledgments}

The authors thank Judy Hannan, RN, MPH; Haley Stolp, MPH; and Hilary Wall, MPH, Centers for Disease Control and Prevention, for their dedication and guidance, and work on the design of this project. The authors also thank Heather Hodge, M.Ed., YMCA-USA, and Alicia Smith, MPH, Association of State and Territorial Health Officials, and the following health centers and health center controlled networks for their dedicated efforts in this project: Affinia Healthcare, St. Louis, Missouri; ARcare, Augusta, Arkansas; Finger Lakes Community Health, Geneva, New York; Hudson River Healthcare, Peekskill, New York; Open Door Family Medical Centers, Ossining, New York; Samuel U. Rodgers Health Center, Kansas City, Missouri; Shawnee Christian Healthcare Center, Louisville, Kentucky; White House Clinics, Richmond, Kentucky; Whitney M. Young, Jr. Health Center, Albany, New York; Kentucky Health Center Network, Mt. Sterling, Kentucky; Missouri Primary Care Association, Jefferson City, Missouri; and HealthEfficient, Albany, New York.

Additionally, the authors would like to thank Curt Bay, $\mathrm{PhD}$ and Aaron Allgood, DO of A.T. Still University of Health Sciences for assistance with statistical analysis and clinical expertise pertaining to hypertension, respectively.

\section{Funding}

This project was funded by US Federal Award Identification Number (US) U8OT00223 from the Centers for Disease Control and Prevention (CDC).

\section{Disclosure}

The authors report no conflicts of interest in this work.

\section{References}

1. Christensen AJ, Howren MB, Hillis SL, et al. Patient and physician beliefs about control over health: association of symmetrical beliefs with medication regimen adherence. J Gen Intern Med. 2010;25 (5):397-402. doi:10.1007/s11606-010-1249-5 
2. Bosworth HB, Powers BJ, Oddone EZ. Patient self-management support: novel strategies in hypertension and heart disease. Cardiol Clin. 2010;28(4):655-663. doi:10.1016/j.ccl.2010.07.003

3. Roumie CL, Elasy TA, Greevy R, et al. Improving blood pressure control through provider education, provider alerts, and patient education: a cluster randomized trial. Ann Intern Med. 2006;145 (3):165-175. doi:10.7326/0003-4819-145-3-200608010-00004

4. Coleman K, Austin BT, Brach C, Wagner EH. Evidence on the chronic care model in the new millennium. Health Aff. 2009;28 (1):75-85. doi:10.1377/hlthaff.28.1.75

5. Control CfD, Prevention. Self-Measured Blood Pressure Monitoring: Actions Steps for Clinicians. Atlanta, GA: Centers for Disease Control and Prevention, US Dept of Health and Human Services; 2014:3-10.

6. Milani RV, Lavie CJ, Bober RM, Milani AR, Ventura HO. Improving hypertension control and patient engagement using digital tools. $\mathrm{Am}$ J Med. 2017;130(1):14-20. doi:10.1016/j.amjmed.2016.07.029

7. Asayama K, Ohkubo T, Kikuya M, et al. Prediction of stroke by self-measurement of blood pressure at home versus casual screening blood pressure measurement in relation to the Joint National Committee 7 classification: the Ohasama study. Stroke. 2004;35 (10):2356-2361. doi:10.1161/01.STR.0000141679.42349.9f

8. Cohen JB, Cohen DL. Integrating out-of-office blood pressure in the diagnosis and management of hypertension. Curr Cardiol Rep. 2016;18(11):112. doi:10.1007/s11886-016-0780-3

9. Uhlig K, Patel K, Ip S, Kitsios GD, Balk EM. Self-measured blood pressure monitoring in the management of hypertension: a systematic review and meta-analysis. Ann Intern Med. 2013;159(3):185-194. doi:10.7326/0003-4819-159-3-201308060-00008

10. Force CPST. Guide to Community Preventive Services-cardiovascular Disease Prevention and Control: Team-Based Care to Improve Blood Pressure Control. Atlanta, GA: US Department of Health and Human Services, CDC; 2012.

11. Jackson SL, Ayala C, Tong X, Wall HK. Clinical implementation of self-measured blood pressure monitoring, 2015-2016. Am J Prev Med. 2019;56(1):e13-e21. doi:10.1016/j.amepre.2018.06.017

12. Cheng C, Studdiford JS, Chambers CV, Diamond JJ, Paynter N. The reliability of patient self-reported blood pressures. $J$ Clin Hypertens. 2002;4(4):259-264. doi:10.1111/j.1524-6175.2002.01042.x

13. Bonafini S, Fava C. Home blood pressure measurements: advantages and disadvantages compared to office and ambulatory monitoring. Blood Press. 2015;24(6):325-332. doi:10.3109/08037051.2015. 1070599

14. Whelton PK, Carey RM, Aronow WS, et al. 2017 ACC/AHA/AAPA/ $\mathrm{ABC} / \mathrm{ACPM} / \mathrm{AGS} / \mathrm{APhA} / \mathrm{ASH} / \mathrm{ASPC}$ NMA/PCNA guideline for the prevention, detection, evaluation, and management of high blood pressure in adults: a report of the American College of Cardiology/ American Heart Association Task Force on Clinical Practice Guidelines. J Am Coll Cardiol. 2018;71(19):e127-e248. doi:10.10 16/j.jacc.2017.11.006
15. Cheng C, Studdiford JS, Diamond JJ, Chambers CV. Primary care physician beliefs regarding usefulness of self-monitoring of blood pressure. Blood Press Monit. 2003;8(6):249-254. doi:10.1097/ 00126097-200312000-00005

16. Carter EJ, Moise N, Alcántara C, Sullivan AM, Kronish IM. Patient barriers and facilitators to ambulatory and home blood pressure monitoring: a qualitative study. Am J Hypertens. 2018;31 (8):919-927. doi:10.1093/ajh/hpy062

17. Logan AG, Dunai A, McIsaac WJ, Irvine MJ, Tisler A. Attitudes of primary care physicians and their patients about home blood pressure monitoring in Ontario. $J$ Hypertens. 2008;26(3):446-452. doi:10.1097/HJH.0b013e3282f2fdd4

18. Muntner P, Shimbo D, Carey RM, et al. Measurement of blood pressure in humans: a scientific statement from the American Heart Association. Hypertension. 2019;73(5):e35-e66. doi:10.1161/ HYP.0000000000000087

19. Viera AJ, Cohen LW, Mitchell CM, Sloane PD. How and why do patients use home blood pressure monitors? Blood Press Monit. 2008;13(3):133-137. doi:10.1097/MBP.0b013e32830263b7

20. Bryant KB, Sheppard JP, Ruiz-Negrón N, et al. Impact of selfmonitoring of blood pressure on processes of hypertension care and long-term blood pressure control. J Am Heart Assoc. 2020;9(15): e016174. doi:10.1161/JAHA.120.016174

21. Pickering TG, Miller NH, Ogedegbe G, Krakoff LR, Artinian NT, Goff D. Call to action on use and reimbursement for home blood pressure monitoring: a joint scientific statement from the American Heart Association, American Society of Hypertension, and Preventive Cardiovascular Nurses Association. Hypertension. 2008;52(1):10-29. doi:10.1161/HYPERTENSIONAHA.107.189010

22. Jones MI, Greenfield SM, Bray EP, et al. Patients' experiences of self-monitoring blood pressure and self-titration of medication: the TASMINH2 trial qualitative study. Br J Gen Pract. 2012;62(595): e135-e142. doi:10.3399/bjgp12X625201

23. Meador M, Hannan J, Roy D. et al. Accelerating use of self-measured blood pressure monitoring (SMBP) through clinical-community care models. J Community Health. 2020:1-12. doi:10.1007/s10900-01900710-0

24. Shimbo D, Artinian NT, Basile JN, et al. Self-measured blood pressure monitoring at home: a joint policy statement from the American Heart Association and American Medical Association. Circulation. 2020;142(4):e42-e63. doi:10.1161/CIR.0000000000000803
Integrated Blood Pressure Control

\section{Publish your work in this journal}

Integrated Blood Pressure Control is an international, peer-reviewed open-access journal focusing on the integrated approach to managing hypertension and risk reduction. Treating the patient and comorbidities together with diet and lifestyle modification and optimizing healthcare resources through a multidisciplinary team approach constitute key features of the journal. This journal is indexed on
American Chemical Society's Chemical Abstracts Service (CAS) The manuscript management system is completely online and includes a very quick and fair peer-review system, which is all easy to use. Visit http://www.dovepress.com/testimonials.php to read real quotes from published authors. 